\title{
El cuidador, rechazo y pérdida del trasplante renal en niños y adolescentes ${ }^{\dagger}$
}

\author{
The caregiver, rejection and loss of kidney transplantation in children and \\ adolescents \\ O cuidador, rejeição e perda do transplante renal em crianças e adolescentes
}

\author{
Luz Viviana Restrepo-Marulanda \\ Ángela María Salazar-Maya ${ }^{2}$
}

\begin{abstract}
Autor de correspondencia*
* Enfermera. Magister en Enfermería de la Universidad de Antioquia. Enfermera Hospital San Vicente Fundación. Correo: vialaya@hotmail.com iD

${ }^{2}$ Enfermera. Doctora en Enfermería, profesora titular de la Facultad de Enfermería de la Universidad de Antioquia y profesora de cátedra de la Universidad CES. Correo: angela.salazar@udea.edu.co.
\end{abstract}

C) Universidad Francisco de Paula Santander Este es un artículo bajo la licencia CC-BY-NC-ND (c) $($ i) $(\Theta)$

\section{Resumen}

Objetivo: Interpretar las categorías cuidador, rechazo y pérdida del trasplante, del estudio Trayectoria del paciente pediátrico con trasplante renal, desde la perspectiva del cuidador. Materiales y Métodos: Investigación cualitativa exploratoria, descriptiva; se realizaron entrevistas semiestructuradas a 14 cuidadoras. Para el análisis se usaron las herramientas de la teoría fundamentada. por medio de la codificación abierta, axial y selectiva. Resultados: La mayoría de las cuidadoras familiares, fueron sus madres, mayores de 30 años. La categoría cuidador la conforman las subcategorías: Conformación del grupo familiar y el reconociendo el cuidador. La categoría el rechazo y la pérdida del trasplante, está conformada por las subcategorías: Presentando rechazo y buscando las causas del rechazo, manejando las complicaciones y los sentimientos que genera el rechazo. Si bien es cierto que existen personas que intervienen en el cuidado, específicamente familiares, también lo es, que debe existir un cuidador que esté siempre a cargo del niño. Por esto, el cuidador es la persona que conoce todo el proceso, es el apoyo, el eje, de él depende todo y siempre debe estar ahí, así el niño crezca. El rechazo y la perdida de trasplante lo vieron como una complicación del proceso y describen sus posibles causas, como las manejaron y los sentimientos que se desencadenaron. Conclusión: Gran parte del éxito del trasplante se le atribuye al cuidador, pues es quien cuida permanentemente del niño y quien guía los cuidados, la condición de ser niños les otorga total dependencia del cuidador familiar.

Palabras clave: Trasplante de riñón; cuidadores; investigación cualitativa; cuidador familiar

$\dagger \quad$ Articulo derivado del trabajo de grado titulado: la investigación: Trayectoria del paciente pediátrico con trasplante renal, desde la perspectiva del cuidador. Para optar al título de Magister en Enfermería de la Facultad de Enfermería de la Universidad de Antioquia.

Recibido: 25 enero 2020. Aprobado: 6 julio 2020

Para citar este artículo/ To reference this article/ Para citar este artigo/

Restrepo-Marulanda LV, Salazar-Maya AM. El cuidador, rechazo y pérdida del trasplante renal en niños y adolescentes. Rev. cienc. cuidad. 2020; 17(3):20-32. https://doi.org/10.22463/17949831.1696 


\begin{abstract}
Objective: Interpret the categories: caregiver; "The rejection" and "the loss of the transplant", from the study Path of the pediatric patient with kidney transplant, from the perspective of the caregiver. Materials and Methods: Qualitative, exploratory, descriptive research; Semi-structured interviews were conducted with 14 caregivers. For the analysis, grounded theory tools were used. by means of open, axial and selective coding. Results: Most of the family caregivers were their mothers, over 30 years of age. The caregiver category is made up of the subcategories: conformation of the family group and recognizing the caregiver. The category The rejection and loss of the transplant is made up of the subcategories presenting rejection and looking for the causes of rejection, managing the complications and feelings generated by rejection. While it is true that there are people involved in care, specifically family members, it is also true that there must be a caregiver who is always in charge of the child. For this reason, the caregiver is the person who knows the whole process, is the support, the axis, everything depends on him, the one who must always be there, even if the child grows up. Transplant rejection and loss are seen as a complication of the process and describe its possible causes, how they are handled and the feelings it generates. Conclusion: Much of the success of the transplant is attributed to the caregiver, since they are the one who permanently cares for the child and who guides the care, the condition of being children gives them total dependence on the family caregiver.
\end{abstract}

Keywords: Kidney transplant; caregivers; qualitative research; family caregiver.

\title{
Resumen
}

Objetivo: interpretar as categorias: cuidador; "Rejeição" e "Perda de transplante", do Estudo em Paciente Pediátrico com Transplante Renal, na perspectiva do cuidador. Materiais e Métodos: pesquisa descritiva, exploratória, qualitativa; Entrevistas semiestruturadas foram realizadas com 14 cuidadores. Para a análise, foram utilizadas as ferramentas da teoria fundamentada. através de codificação aberta, axial e seletiva. Resultados: a maioria dos cuidadores familiares era mãe, com idade superior a 30 anos, sendo a categoria cuidador composta por subcategorias: conformação do grupo familiar e reconhecimento do cuidador. A categoria Rejeição e perda de transplante é composta por subcategorias que apresentam rejeição e buscam as causas da rejeição, gerenciando complicações e sentimentos gerados pela rejeição. Embora seja verdade que existem pessoas envolvidas no cuidado, especificamente membros da família, também é verdade que deve haver um cuidador que esteja sempre encarregado da criança. Por esse motivo, o cuidador é a pessoa que conhece todo o processo, é o suporte, o eixo, tudo depende dele, que sempre deve estar lá, mesmo que a criança cresça. A rejeição e perda do transplante o vêem como uma complicação do processo e descrevem suas possíveis causas, como são tratadas e os sentimentos que gera. Conclusão: Grande parte do sucesso do transplante é atribuída ao cuidador, uma vez que é ele quem cuida permanentemente da criança e orienta o cuidado, uma vez que a condição de ser criança lhes dá total dependência do cuidador familiar

Palavras-chave: Transplante de rim; cuidador familiar; pesquisa qualitativa. 


\section{Introducción}

ISSN-PRINT

1794-9831
La enfermedad renal crónica se caracteriza por un filtrado glomerular disminuido y/o presencia de marcadores de daño renal: albuminuria aumentada, anomalías del sedimento urinario, electrolíticas u otras debidas a trastornos tubulares; o detectadas histológicamente, o estructurales detectadas con pruebas de imagen (1).

Poco se conoce en nuestro medio sobre la incidencia y prevalencia de la enfermedad renal crónica (ERC) en los niños, ya que los valores reales se subestiman o no se registran. Los registros europeos muestran incidencias alrededor de 10-12 pacientes por millón de población pediátrica (ppmp) y prevalencias alrededor de 59-74 ppmp. (1).

Entre las causas de la ERC están la raza afroamericana, las anomalías estructurales, las enfermedades renales quísticas y hereditarias, las vasculares y las glomerulopatías primarias o secundarias (1).

Para atender esta patología existen distintas alternativas de tratamiento cuando no se puede llevar un tratamiento conservador, entre ellas están la hemodiálisis, diálisis peritoneal y trasplante, siendo esta última la de elección porque proporciona mejor calidad y expectativa de vida (2).

Los estudios relacionados sobre el tema generalmente han sido realizados por disciplinas diferentes a la enfermería en su gran mayoría en temáticas sobre: farmacovigilancia (3), supervivencia (4), adherencia terapéutica (5), sobrevida (6), revisiones históricas (7) entre muchas otras temáticas.

Por tanto, para la enfermería es importante comprender la trayectoria (situaciones, vivencias, condiciones, experiencias a través del tiempo) y la realidad de los niños con trasplante renal y de sus cuidadores familiares) y así obtener un conocimiento profundo acerca de cómo se afronta este cambio desde todos los ámbitos; permitiendo un mayor acercamiento y empatía con las personas que atraviesan por esta situación. El cuidador se define como "la persona que, siendo cónyuge, compañero o compañera permanente, pariente hasta el quinto grado de consanguinidad, tercero de afinidad, primero civil o que, sin tener ningún tipo de parentesco con la persona con dependencia de cuidado, es apoyo permanente para las actividades básicas e instrumentales de la vida diaria, sin recibir contraprestación económica por su asistencia y por su labor dedicada al cuidado de otra persona" (8).
Por todo lo anterior se planteó como pregunta de investigación: ¿Cómo es la Trayectoria del paciente pediátrico con trasplante renal, desde la perspectiva del cuidador? y como propósito para el presente artículo: Explorar las categorías: cuidador; el rechazo y la pérdida del trasplante.

\section{Materiales y Métodos}

Se realizó una investigación cualitativa exploratoria, descriptiva (9) y se utilizó para el análisis las herramientas de la teoría fundamentada (10), la cual emerge de la teoría sociológica del interaccionismo simbólico (11), que enfatiza en la creación de significados compartidos formados en los procesos de interacción social, significados que se transforman en "realidad" para los individuos que los producen.

Es a través del estudio de las interacciones de la vida cotidiana, donde se construyen los significados, los cuales emergen de los resultados a partir del análisis directo de los datos y no de conceptos y teorías preconcebidas (11). Su valor radica en la descripción y en particular en el desarrollo de temas en un contexto específico.

Inicialmente los participantes se identificaron en una institución de cuarto nivel de la ciudad de Medellín Colombia y recomendaron conocidos que habían experimentado el fenómeno de interés, los cuales fueron contactados por la investigadora; se les invito a participar en el estudio, y así sucesivamente se les preguntó si conocían a otras personas que vivieron esta situación (12). Los criterios de inclusión fueron: Cuidador de niño o adolescente a quien le fue realizado trasplante renal, mayor de edad, tiempo de acompañamiento mínimo de 6 meses. El instrumento de recolección de la información fue la entrevista semiestructurada, la cual se audio grabó y posteriormente se transcribió. Con una pregunta orientadora: ¿podría describirme como ha sido el proceso de trasplante renal desde el diagnóstico hasta como se encuentra ahora?. Además, se diligenció un formato de datos sociodemográficos del cuidador y otros relacionados con el trasplante del niño y/o adolescente. La entrevista se llevó a cabo en el lugar elegido por el cuidador, como la casa, el lugar de trabajo, el hospital; estas se agendaron previamente y el tiempo promedio de duración fue de 45 minutos.

Se utilizó muestreo por conveniencia y el teórico. El primero hizo referencia a la selección de los sujetos, dada la accesibilidad y proximidad que hayan vivido con el fenómeno de interés (12) y el segundo, muestreo teórico, hizo referencia a 
la codificación de los datos, lo que permitió la emergencia de categorías/subcategorías que generaron conceptos a través del método de comparación constante de cada acontecimiento, o suceso; lo que permitió analizar como variaron las categorías en términos de sus propiedades y dimensiones y así tomar decisiones para hacerlas más densas (10). La Saturación teórica se alcanzó cuando no hubo datos nuevos importantes emergiendo de las categorías, y cada una de ellas estuvieron desarrolladas en términos de sus propiedades y dimensiones, lo que demostró que las relaciones entre ellas estuvieron bien establecidas, densas y validadas (10).

El análisis de datos se realizó desde el comienzo hasta el final de la investigación, mediante el método inductivo por la descripción de significados, experiencias, anécdotas. Se utilizaron las herramientas de la teoría fundamentada, que comprendió tres formas de codificación: Codificación abierta; en este proceso analítico, se identificaron los conceptos, sus propiedades y dimensiones (10). Se realizó un análisis minucioso, donde el texto se desglosó línea por línea, se distinguieron las unidades de sentido (significados para el estudio), a las cuales se les asignó un código, lo más cercano al lenguaje de los participantes. Los datos se desagregaron en partes discretas, se examinaron detenidamente y se compararon con el fin de encontrar semejanzas y diferencias. Se buscó generar categorías y sus propiedades, para posteriormente determinar la variación en su rango dimensional (10).

La codificación axial permitió relacionar categorías y subcategorías siguiendo las líneas de sus propiedades y dimensiones, observando cómo se entrecruzaban y se vinculaban (10), es decir que se compararon ciertas características comunes; también conceptos que representaron fenómenos. En la codificación selectiva, se seleccionó la categoría central, que relacionó todas las categorías, se contrastaron estas relaciones con los datos y se completaron las categorías que necesitaban mayor precisión y/o desarrollo, lo que permitió integrar, refinar y pulir las categorías (10). El primer paso fue identificar y seleccionar el fenómeno central relevante para el estudio, que ayudó a responder la pregunta de investigación. El segundo paso consistió en examinar y seleccionar las subcategorías relacionadas con el fenómeno, estas indicaron en qué condiciones ocurrió el fenómeno, cuándo y cuáles fueron las consecuencias y cuáles fueron sus propiedades y dimensiones (10). Para este caso, la categoría central, fue un código in vivo como factor común y de unión de todas las categorías, titulada: Es muy triste saber que uno tiene la vida así, colgadita de un pelito por un trasplante. C3 Esta integración se dio con el pasar del tiempo y con el inició el análisis y sólo terminó con el escrito final (10).

Para esta investigación se consideraron todos los aspectos éticos de acuerdo con las recomendaciones internacionales de la Investigación Biomédica en Seres Humanos, establecidas por el Consejo de Organizaciones Internacionales de Ciencias Médicas (CIOMS) (13), en colaboración con la Organización Mundial de la Salud (OMS), Ginebra, 2002 y la normatividad nacional. A su vez, se acogió a los siete requisitos propuestos por el autor Ezequiel Emanuel (14): valor social, validez científica, selección equitativa de los sujetos, razón riesgo/beneficio favorable, evaluación independiente, consentimiento informado y respeto por los sujetos inscritos con el fin de asegurar que sean tratados con justicia y dignidad mientras contribuyen al bien social.

También se tuvo en cuenta en el presente estudio, lo planteado en la Resolución 008430 de 1993 (15), donde se referencian los artículos relacionados con la identificación del riesgo para las personas que participan de una investigación, con el fin de proteger sus derechos y evitar así que fueran vulnerados. Para este caso, se consideró la investigación con riesgo mínimo, ya que, a pesar de que no se realizó ninguna intervención o modificación con intención en los participantes, la investigadora reconoció que pudieron presentarse en algún momento, molestias y/o alteraciones "de tipo moral, emocional y psicológico" al momento de llevarse a cabo el registro de datos en las entrevistas (16), que en lo posible se evitaron o redujeron por la investigadora, especialmente dando tiempo, acompañamiento, libertad e información a la persona para tramitar dichos asuntos de manera adecuada. La investigación estuvo avalada por el comité de ética de la Facultad de Enfermería de la Universidad de Antioquia, acta CEI-FE 2016-49 y el Comité de Ética del hospital San Vicente Fundación, el 17 de mayo de 2017 en Acta N 08-17.

\section{Resultados}

Participaron 14 cuidadores de niños y/o adolescentes a quienes se les practicó trasplante renal. Trece de ellos eran madres, todas de sexo femenino, con un rango de edad entre 30 y 61 años, en su mayoría separadas, con predominio de escolaridad primaria, en mayor porcentaje trabajadoras independientes, de estrato socioeconómico 2, con un régimen de salud contributivo, la mayoría 


\section{Luz Viviana Restrepo-Marulanda, Ángela María Salazar-Maya}

residentes en municipios de Antioquia.

ISSN-PRINT

1794-9831

E-ISSN 2322-7028

Vol. 17 No. 3

Sep - Dic 2020

Cúcuta, Colombia
En los niños trasplantados el rango de edad fue de 5 a 17 años, de ellos 9 de género femenino y 5 masculino; siete de ellos cursaban básica primaria, seis básica secundaria, y uno en preescolar. Tiempo de trasplante de 2 a 5 años y con 3 hospitalizaciones la mayoría en el transcurso del postrasplante al presentarse complicaciones por rechazo, debido al aumento de la creatinina, por infecciones urinarias y por falta de medicamento. De los 14 participantes, 5 presentaron rechazo y 2 presentaron rechazo con posterior perdida del injerto

De la investigación emergieron las siguientes categorías: Descubriendo la enfermedad; alternativas de tratamiento: diálisis; llegando el trasplante; continuando el proceso; tratamiento; el rechazo y pérdida del trasplante; el cuidador. De las cuales se interpretan el rechazo y perdida del trasplante y el cuidador.

\section{EI cuidador.}

Si bien es cierto que existen personas que intervienen en el cuidado, específicamente familiares, también lo es, que debe existir un cuidador que esté siempre a cargo del niño. Para efectos de la investigación, el cuidador es definido como la persona que conoce todo el proceso de este, es el apoyo, el eje, de él depende todo, el que siempre debe estar ahí, así el niño crezca. Es quien conoce todo el tratamiento, administra los medicamentos, sabe los cuidados que debe tener, hace seguimiento continuo, tiene una comunicación permanente con el médico y realiza los trámites relacionados con citas, autorizaciones y obtención de los medicamentos.

En otras palabras, es la persona que se preocupa diariamente por el bienestar del niño; por tanto, debe contar con unas características específicas para poder brindar un cuidado adecuado, como estar psicológica y físicamente sano, para que así pueda transmitir lo mejor y, por ende, estar en condiciones para llevar a cabo el cuidado. Coincidiendo también que idealmente el cuidador sea la mamá, ya que es quien siente y le duele todo el proceso. El cuidador vive, siente y sueña por brindar lo mejor, para mantener en un óptimo estado de salud al niño. A esta categoría la componen las siguientes subcategorías:

- Conformación familiar. Hace referencia a como está conformado el núcleo familiar del niño, quiénes conviven con él y quiénes en algún momento hicieron parte de este. Tam- bién describe los cambios que puede tener esta conformación en el transcurso del tiempo. Como refieren madres cabeza de familia: Soy madre cabeza de familia...y me mantengo afuera...Ella está solita. Porque el papá tampoco vive con ella porque yo soy separada hace 15 años de él. Cl.

La separación de los padres, y la muerte también está presente:

Hace un año me había separado del papá, pues el papá se fue y lo había dejado. Y él vio muchas cosas, y a él le impactó mucho, el niño vio que el papá estaba viviendo con la muchacha... el niño todavía dice, si llevábamos una semana [de separados] porque tenía que vivir en la casa con ella, él si los abandonó mucho, cuando nos dejamos. $\mathrm{C4}$

Todo el tiempo he sido Dios conmigo y yo para ellos, porque el papá del niño falleció cuando él estaba muy pequeñito, no tenía ni un año de nacido. A los dos años que me conocí con un muchacho fue que tuve al otro niño. Yo tuve mi niño sola y levanté al otro. Yo me pegué de Dios... yo tengo cuatro hijos, y yo con esos cuatro acá, ya trabajaba por dias, mi hermanita me ayudaba. C14

-Reconociendo el cuidador. Hace parte del significado que se le da a este y a su intervención en todo el proceso. En cierta forma se le atribuye la responsabilidad del éxito del trasplante, ya que es el apoyo y quien cuida permanentemente del niño y quien guía los cuidados. Reconoce que es un proceso muy duro por el desgaste de las hospitalizaciones, la realización de trámites, los permisos que se deben pedir en el trabajo, lo inconvenientes con la pareja por la realización de trámites.

También se afirma que es un proceso que debe ser llevado a cabo por la mamá del niño, ya que esta es quien conoce al niño y a quien le duele todo lo que le pase. Reconocen que se puede contar con dos cuidadores, por ejemplo, las abuelas, las cuales permiten complementar el cuidado del niño, al no contar con la disponibilidad de los padres; ellas se encuentran preparadas para el manejo del niño y cuentan con disponibilidad. Gran parte del cuidado del niño, en especial de acuerdo con la edad, depende del cuidador y son muchos los asuntos de los que debe estar pendiente. Los que tienen pareja o una familia conformada, se ponen de acuerdo para realizar las diferentes tareas en pro del cuidado del niño lo que evita el 
agotamiento; pero también los padres trabajan y es la hija la que cuidaba del niño. Igualmente se debe tener en cuenta que el bienestar del cuidador es indispensable para el cuidado del niño trasplantado:

Es importante que el cuidador psicológicamente este sano, porque eso agota...tanto el cuidador como el paciente deben estar muy bien nutridos... el cuidador tiene que estar muy bien... si una persona está estresada o preocupada se transmite, eso es una energía que se transmite... nosotros hemos tratado de estar muy sanos mentalmente. C10

También refieren que parte de ese bienestar es alternar el cuidado del niño:

La persona que va a estar cuidando al niño va a estar agotada. Entonces va a estar papá y mamá y se van a estar alternando... Si uno se trasnocha, el otro descansa, y psicológicamente deben de estar bien. C10

Todos necesitamos quién nos guie y nos apoye en muchas cosas ... y siempre todo el mundo necesitamos apoyo, nadie es capaz de llevar una situación buena o mala en la vida, siempre necesitamos quién nos diga si se puede o está mal o está bien. Ella y yo somos como una base o como una mesa, si falta una pata, se cae. C11

-La importancia de ser cuidador. Su nombre lo dice, es el valor que se le da al cuidador, ya que es quien hace que no se pierda el trasplante por sus cuidados, porque estas personas dependen totalmente de él. Es el responsable de la consecución de los cuidados, su importancia radica en estar ahí siempre. Sin embargo, se afirma que es la mamá la que debe ser la cuidadora, por el lazo que se genera desde el nacimiento, porque ella es quien siente realmente lo que le sucede al niño:

Muchas veces ellos pierden los riñoncitos, no es ellos, es uno... es muy importante, como le dijera yo, si ser responsable, porque es que ella verá, uno serelaja. Unotambién tiene que ponermucho desu parte en eso, no solo ellos, si no uno también. C5.

Lo hemos comprobado...el lazo que hay entre hijo a mamá es como un hilo que hace que él, salga adelante más fácil...no sé cómo explicarle, pero desde que el nació desde que yo lo tuve, es como un lazo. Yo siento que cuando yo estoy, el niño es aliviado totalmente...es más como el amor que los dos sentimos y siento que de alguna forma él ha tratado de salir adelante...él se siente como tranquilo como en un confort como que no le falta nada...pero más que el cuidado, es más como el amor que los dos sentimos. El amor de mamá es un lazo más allá de lo físico...es algo como interno que hace que los niños sientan ese cuidado y eso los hace aliviar más fácil...es fundamental el amor de mamá, no es lo mismo que el papá. $C 10$

-Intervenciones de la familia y de otras personas en el cuidado. En el cuidado del niño no solo intervienen los cuidadores, también otras personas, debido a que aquellos tienen otras ocupaciones que los obligan, en algún momento, a dejar los niños a cargo de otras personas. Generalmente estas personas tienen conocimientos de los cuidados y/o están entrenadas en el manejo del niño trasplantado. Describen el papel que desempeña cada uno de los miembros definidos por afinidad de sentimientos, como el acompañamiento que hace la familia al niño y al cuidador en todo el proceso del cuidado, con el fin de que se sientan apoyados y respaldados. Intervienen en el cuidado: los abuelos, tíos, hermanas, y hasta los profesores, hacen parte del cuidado y tienen conocimientos de este:

Yo tengo mis hermanos, hay un hermano que siempre ha estado ahi pero nunca había estado tan pendiente y en las últimas hospitalizaciones, él me dijo yo voy y la cuido, vaya descanse usted ya lleva mucho tiempo. Me senti apoyada, pues mi hermano al que ella siempre le dice papá, para ella eso era muy bien, igual ellos siempre han estado ahí...C13

A la niña me la dejan ir al baño, inclusive, para que no vaya a los baños del colegio, utiliza el de los profesores. Todos los profesores son conscientes de que la niña, tiene muchas prioridades, por ejemplo, cuando estaba muy pequeña, si se le mandaba la droga y la profesora se encargaba de dársela a la hora que era. Ellas colaboran mucho con eso. C3

\section{El rechazo y la pérdida del trasplante.}

Es una de las complicaciones del trasplante, en la cual está en riesgo el niño y el injerto (riñón trasplantado). A su vez, describe las múltiples causas por las que esto puede pasar, el manejo de dicha complicación y los sentimientos y emociones que genera en el niño y el cuidador el atravesar por esta situación. Es un momento que puede convertirse en un retroceso, en pensar tener que volver a la vida antes del trasplante, volver a diálisis y perder todo el tiempo que han invertido antes y después del trasplante. En la mayoría de los casos se desconocía que el rechazo no era sinónimo de pérdida del trasplante, ya que no necesariamente cuando hay rechazo, hay pérdida del injerto; pue- 
de convertirse en una alerta, un indicio de que algo no está funcionando adecuadamente, ya sea relacionado con el seguimiento del tratamiento, con la entrega de medicamentos, con la edad del niño/adolescente, con el cuidado de los aspectos relacionados con la dimensión emocional del niño. También hace referencia a las complicaciones relacionadas con el rechazo y a los múltiples tratamientos por los que debe pasar el niño, con el fin de no perder el trasplante, pues en algunas ocasiones estos no son exitosos. A esta categoría la componen las siguientes subcategorías:

- Presentando rechazo - buscando causas del rechazo. En los momentos en que el niño presenta alteraciones de salud o en los exámenes de rutina y en los que está, o puede estar en riesgo la vida y el trasplante. Define los signos y síntomas característicos del rechazo. Describen el posible origen del rechazo, sea relacionado con la administración de medicamentos, cambio en la prescripción.

Después del trasplante ha tenido tres rechazos. Yo no me sé el nombre, pero el médico me dijo que habia 3 rechazos del riñón trasplantado, que ella ya le habian dado los tres, pero sin embargo está ahí... C13

Hizo rechazo, en el 2017 tuvo como el rechazo... Le hicieron un examen y era porque las células de él y las del donante no eran compatibles con él...El niño era compatible, porque a ellos le hacen unos exámenes, pero no en esta parte de las células. C12

Describen también como la preadolescencia puede ser un factor predisponente a la no adherencia al tratamiento, ya que en esta etapa de la vida se preocupan más por la imagen corporal.

Unas veces se tomaba los medicamentos otras veces no. Ella pasó de la escuela a colegio, ya es más diferente. Ella no contaba, pero yo digo que se sentía mal porque a ella le tocaba hacerse cateterismos, le tocaba tomar medicamentos. Ella se los llevaba en el bolso y yo le decía, los medicamentos, y ella decía, se me olvidó. Yo digo que ella se sentía mal ir a hacerse los cateterismos, entonces se descuidó. C4

Otra posible causa del rechazo identificada se puede relacionar con la entrega de medicamentos:

Fue porque la EPS nos daba los medicamentos genéricos, me dio medicamentos genéricos y yo se los daba a ella porque yo no sabía nada, entonces ya empezó el rechazo de riñón. Y la traje al hospital...Si me los daban bien. Pero antes no eran los de alto costo, si no los genéricos, los más baratos, y yo se los suministraba. $\mathrm{C} 7$

Ella estaba tomando 6 gramos de tacrolimux y la doctora le cambió a 5 gramos, ella tomaba tres por la mañana y tres por la tarde, y le cambió a 5 gramos cada 24 horas... y el que a mi me habian devuelto era de un miligramo, no me lo entregaron porque no lo conseguían, se demoraron más de 15 días para conseguirlo, entonces yo empecé a darle, ese que me habian devuelto, no fijándome que era de un miligramo, y le estaba dando nada.C9

O los cambios del medicamento:

El primero empezó a tomar tacrolimux pero el convencional y ese como que el cuerpo no lo asimiló bien y ya después se lo cambiaron a XL... Se notó que asimilaba mejor el XL por los exámenes, variaban mucho de un medicamento o a otro, entonces se le cambió a XL y ya lo asimiló muy bien. $\mathrm{C} 12$

Dificultades con los cuidados. Al cuidador le queda imposible hacer seguimiento del tratamiento:

No pude (cuidador) estar ahi todos esos días, ahi constante, dándole la droga, le hice unos exámenes y todos le salieron malos. C5.

O la misma depresión:

Él no se tomaba los medicamentos por la tristeza que tenía. Él se sentía aburrido. Yo le pregunté a él, papi a ti te afectó que tu hermanita no hubiera venido en el cumpleaños y a él se le aguaron los ojos y me dijo que sí. Yo le dije, pero es que usted no puede apegarse a eso, porque debes de entender, que ella ya tiene su hogar, tienes sus hijos, siempre que usted cumplía años, ella era la que lo sacaba y se lo llevaba, y usted no debe de hacer que el riñón se enferme por esa bobada, usted tiene que tener conocimiento porque usted ya tiene 14 años, y usted no es un niño, usted ya va siendo un joven. Ese embarazo le quedó muy duro, él con la hermanita podía decir que más que yo, porque como ella lo cuidó tanto y ella se casó, entonces el día que cumplía años la hermanita iba y lo buscaba, lo llevaba a comer, a paseos. Este año no lo llevó a ninguna parte...los exámenes se le subieron, unos se le bajaron y fue que empezó con la hospitalización... Tenía que haberse tomado 3 pastillas...Apenas se habia tomado una, la del lunes, la del martes no se la tomó, y la del miércoles no se la había tomado. C14

-Manejando las complicaciones del rechazo. Rev. cienc. cuidad. 2020;17(3):20-32 
Según los métodos indicados por el médico y llevados a cabo con el fin de contrarrestar el rechazo. Muchas alternativas de manejo: plasmaféresis, cambio de medicamento y administrando otro, unos pocos exitosos:

Habia que hacerle unas plasmaféresis y que eso tocaba mediante un catéter...Eso es bien dificil... Tocaba conectarla a una máquina más o menos una hora...Inició tres veces por semana, luego dos veces por semana y si, pues gracias a Dios la mejoría sí, pero. Ella duró harto en ese tratamiento y tuvo complicaciones. Duró de febrero hasta agosto. Se infectó ese catéter entonces tocó hospitalizarla para ponerle otro catéter fue. súper feo. fue bien dificil ese proceso $\mathrm{C} 8$

Empezó a hacerle tratamiento con la gamaglobulina un medicamento intravenoso...Ya es diferente, no está el riesgo de infección de tener ese catéter ahí...Empezó con tratamiento en septiembre de 2016...Desde el primer mes ya se le empezó a ver que si le funcionaba, que si era lo que necesitábamos...Empezó cada 20 días más o menos como unos tres, cuatro meses y ya luego cada mes y en diciembre se lo colocamos de ese 2016 y ya se aguantó 5 meses. C8

Le hicieron una biopsia y le pusieron muchos medicamentos y ya gracias a Dios salimos de eso...A mi me dijeron que el tratamiento que le iban a hacer a ella era demasiado fuerte como cuando la trasplantaron, le pusieron timoglobulina, durante 3 días, que era fuerte, y no le sirvió, entonces se la subieron a 5 días, la doctora me dijo la vamos a aumentar a 5 dias, si este tiempo no reacciona, le tienen que hacer otra biopsia para saber qué vamos a hacer...Como que con los 5 días tuvo gracias a Dios. C7

- Sentimientos que genera el rechazo en el niño y la mamá. Se desencadenan sentimientos y emociones por parte del niño y el cuidador, específicamente por la incertidumbre y el miedo a las complicaciones asociadas al rechazo y las consecuencias de este para la vida:

La doctora me dijo, mira lo que pasa es que vimos que habia un rechazo. Para mi fue como ahi voy a volver a lo mismo voy a volver atrás y fue muy duro... Yo pensaba, que cuando a mi me dijeron rechazo la primera vez, ya ella perdía el riñón que él iba para diálisis otra vez. C13

Sentí que era lo mismo, lo mismo de antes, lo mismo que al principio... Otra vez a la misma rutina... Otra vez la diálisis...Otra vez se puso igual, que la presión alta. C14

\section{Discusión}

Como lo muestran los resultados del estudio uno de los aspectos fundamentales en el cuidado del niño, es la conformación familiar, ya que quienes conviven con él puede afectar el transcurso del trasplante pues la familia es la red de apoyo más cercana para el niño trasplantado. Así mismo lo relatan Medellín y Carrillo (17), la familia cumple su función protectora y ayuda en las situaciones difíciles del niño, además, su adecuado funcionamiento resulta en bienestar. De allí que sea tan importante el grupo de personas que conforman esa red primaria de apoyo y sobre todo la representación que tienen frente a la persona que cuidan.

Como lo refieren Goreti et al., (18), las familias perciben cambios. Algunas comentaron que, finalmente, después del trasplante podrían cuidarse mejor, regresar al trabajo, considerando que, antes del procedimiento, la principal preocupación era cuidar al niño/joven, incluso descuidar el cuidado con su propia salud.

La manera como el niño se adapta y afronta la enfermedad depende en gran parte de la dinámica de funcionamiento de todos los miembros de la familia y de los recursos personales y familiares disponibles para afrontar el problema (17). La armonía familiar, se podía ver reflejada en el adecuado manejo del niño y, por ende, en su adaptación al transcurso de todo el proceso.

En algunos casos se encontraron conflictos familiares por lo que fue difícil distribuir las tareas para el cuidado del niño, lo que ocasionó inconvenientes en la convivencia de pareja y por supuesto generaron cambios en el comportamiento del niño. Dicen los autores que los progenitores de niños enfermos suelen ser descritos como sobreprotectores o restrictivos y las familias suelen tener conflictos y dificultades para tomar decisiones. Con el pasar del tiempo la experiencia puede ser elaborada y promover en algún grado la comunicación familiar y el apoyo mutuo, lo que lo ayuda a afrontar sus problemas (17).

Seguido a esto, las responsabilidades del cuidador familiar son múltiples y demandan tiempo, pues el cuidador quien tuvo que estar siempre pendiente de todo, pues los niños pequeños requirieron toda su atención por su condición. Sin embargo, en el caso de niños más grandes o ado- 


\section{Luz Viviana Restrepo-Marulanda, Ángela María Salazar-Maya}

lescentes también requieren intervención y cuidado a pesar de su edad, necesitan reforzar las conductas constantemente. Cercano a lo que dice DePasquale (19), el seguimiento constante de la toma de medicación por parte de un acompañante o cuidador reduce el mayor tiempo de errores posibles. Los miembros de la familia participan en una multitud de actividades esenciales, algunas de las cuales incluyen la administración de medicamentos; supervisar el cumplimiento del tratamiento; ayudar con las actividades habituales y la entrega del tratamiento; estas actividades benefician al niño/adolescente y a proveedores de salud por igual (19).

Otro aspecto fundamental, que los mismos cuidadores describieron, es el deber de estar psicológicamente bien, pues este estado se transmite al niño y, por el contrario, si está preocupado o estresado también. Así, el bienestar del cuidador fue indispensable para que el cuidado del niño trasplantado fuera eficiente. Similar a lo que dicen Medellín et al, las evidencias indican que el funcionamiento de toda la familia y el estado emocional de los padres y madres son aspectos fundamentales en el bienestar emocional y la adaptación del niño a la enfermedad y trasplante (17). Así mismo, Rodríguez y Fonseca (20), refieren que la salud mental de los padres y madres, por ejemplo, es un aspecto extremamente importante ya que los niños/adolescentes dependen de ellos y necesitan de sus cuidados y protección para sobrevivir. Si los progenitores presentan problemas psicológicos y no están preparados para cuidar de sus niños de la manera requerida, se esperarán efectos negativos en la salud de los mismos (20).

En el estudio los niños fueron cuidados por sus madres, solo uno fue cuidado por sus abuelas materna y paterna. A ellas era a quienes realmente les dolía todo lo que pasaba con el niño y quienes debían asumir la responsabilidad, ya que otra persona no lo iba a realizar de la forma que debía ser. Así lo relatan Tejada et al., (21), la persona que más se implica en los cuidados es la madre. La mayoría de los niños/adolescentes tienen supervisión de la toma de medicación por parte de un cuidador, que habitualmente es la madre. Además Fernández et al., (22) relatan de lo fundamental que es su papel en los cuidados, los padres de los receptores pediátricos y sus familiares proveen cuidado, abordan las cuestiones de rechazo y los efectos adversos de los agentes inmunosupresores

Como lo refiere Pinto (23), en el caso de los niños, las madres se doblegan ante sus hijos, porque "el amor de madre prima sobre todos los principios". Ella asume la mayor parte de la responsabilidad de los cuidados. En el caso de los padres, ellos delegan todas las funciones a las madres, puesto que las consideran más aptas y las sienten más preparadas para cuidarlos. De hecho, ellos manifiestan y demuestran un sentimiento de impotencia que les impide, en la mayoría de los casos, colaborar en el cuidado de sus hijos (23). Similar a lo que dice la autora, algunos padres estuvieron pendientes del niño, aunque no asumieron el rol de cuidador como tal, porque la mayoría estuvieron ausentes.

No obstante, el papel que jugó la familia y personas cercanas al niño fue muy importante, pues estos en cierta forma aportaron al cuidado, mediante el apoyo en momentos difíciles. A su vez, los profesores en la escuela apoyaron la administración de medicamentos, les proveyeron los recursos y tiempos necesarios. Igualmente lo relata Davis (24), el apoyo percibido por la familia o entorno del niño juega un papel fundamental en la responsabilidad ante la toma de medicación. Por lo tanto, el trasplante de un niño tiene una influencia sustancial en la vida diaria de la familia. Cabe resaltar que el apoyo familiar no solo fue visto en relación con la administración de medicamentos, si no con todo lo que comprende el cuidar, con todas sus dimensiones evidenciadas a través del tiempo.

Por otro lado, una de las grandes problemáticas encontradas fue el rechazo y la pérdida del trasplante, donde varios de los cuidadores relataron que el niño ya había sido trasplantado y que había perdido el trasplante y otros relataron que habían tenido varios episodios de rechazo. En la cual se evidenciaron múltiples causas, como la respuesta inmunológica al trasplante, las relacionadas con la entrega de medicamentos, el cambio de medicamentos, la preadolescencia, el seguimiento del tratamiento, las complicaciones del rechazo y las emociones generadas frente al rechazo. Si bien los niños presentaron rechazo por causas inmunológicas, para este estudio, no fue la causa principal. En cuanto a las demoras, la no entrega de medicamentos, y en algunos casos el suministro del medicamento que no correspondía, generó alteraciones en las que se llegó al rechazo, consecuente con lo que dice Agredo et al.,(25), los primeros reportes mostraron que la suspensión de los esteroides se asoció a rechazo agudo en más de $50 \%$ de los casos.

Igualmente, Carrillo et al., (26), refieren que el sistema de salud, también puede ser un obstáculo al no autorizar los medicamentos o los exámenes 
y procedimientos que el niño requiere en ese momento, lo cual interfiere en el bienestar del niño.

Dentro de las causas más relevantes relacionadas con el rechazo, fue la preadolescencia, ya que se determinó que fue un factor predisponente a la no adherencia al tratamiento, pues en esta etapa de la vida, las preocupaciones pasaron a ser más por la imagen corporal que por el seguimiento del tratamiento. Otros aspectos como la transición de la escuela al colegio generaron descuido en el seguimiento del tratamiento. Refirieron que en algunos casos olvidaron tomar sus medicamentos o no se realizaron los cateterismos. Durante la adolescencia las repercusiones de una enfermedad crónica también son significativas; Navarrete et al (27), refieren que en esta etapa del ciclo vital, caracterizada por ambivalencia y contradicciones en cuanto al comportamiento, la apariencia física y la identidad personal se ven afectadas por el reto de mantener la salud y el bienestar. Esto representa un factor de estrés adicional, lo cual genera alteraciones en la autoimagen, factor que provoca aislamiento, así como ajustes en la cotidianidad y en su entorno social, con la consecuente alteración de su rol.

Para el adolescente enfermo, por ejemplo, es notable el efecto que causa en su bienestar la inasistencia a las clases, la disminución en el tiempo compartido con sus pares, el ser rechazado y considerado como discapacitado dentro de su principal entorno social. Semejante a lo que dice el autor, el aislamiento social y la no ingesta de los medicamentos, sobre todo en las mujeres, fueron asociados al sentimiento de vergüenza que les generaban las transformaciones corporales provocadas por la enfermedad y el tratamiento (27). A esto se suman las consecuencias para la autoimagen y el efecto que trae el uso de medicamentos, dispositivos y las cicatrices que disminuyen su atractivo físico, aspectos que pueden ocasionar falta de adherencia al tratamiento (23).

Barra y Morillo (28), refieren que factores como los sociodemográficos, los relacionados con la persona, el tratamiento, la propia enfermedad, el sistema de salud y el mismo equipo de salud afectan en la adherencia al tratamiento. Así mismo the American Society of Transplantation (29) refiere que el incumplimiento en la adherencia al tratamiento está presente en la adolescencia, por lo que se debe dar atención especial, ya que pueden fallar en la adherencia al tratamiento, lo que puede repercutir en la sobrevida del injerto a largo plazo.

Pues, uno de los factores más importantes que influyen en este problema es la irregularidad o https://doi.org/10.22463/17949831.1696 suspensión de la toma habitual de los medicamentos. Muchos adolescentes refieren suspender el tratamiento para evitar los efectos cosméticos de los fármacos inmunosupresores, en particular de la ciclosporina y los esteroides que genera la aparición de acné, hirsutismo, facies leoninas, hipertrofia gingival y obesidad, por esto se ha sugerido que en este grupo de edad se deben emplear otros medicamentos como el tacrolimus y el mofetil micofenolato (27).

Otra causa relevante relacionada con el rechazo que refiere the American Society of Transplantation fue el aspecto emocional, en algunos casos, el niño presentó conductas, como tristeza, decaimiento, negatividad con el tratamiento, rechazo e incumplimiento de este, que estuvieron asociados y relacionadas con inconvenientes familiares, cambios en el entorno familiar etc. Ocurrió, por ejemplo, que el niño no se tomó los medicamentos por la tristeza que tenía. Comparable a lo que dicen los autores, que exponen un caso clínico detectado en consulta de trasplante renal donde el enfermo dejó de tomar toda la medicación prescrita debido a la influencia de circunstancias externas (29). Lo que permite inferir, que el rechazo no se da simplemente por causas clínicas, existen muchas otras causas derivadas de este evento, que en muchos casos pueden pasar desapercibidas o desconocerse y no brindarle importancia requerida, ya que pueden ser muy influyentes a la hora de seguir el tratamiento.

Respecto a los sentimientos que generó el rechazo y en algunos casos la pérdida del trasplante, se encontró que este momento lo asumieron como que tenían que volver a lo mismo, volver atrás, volver a diálisis, y a la vez, la incertidumbre y el miedo a las complicaciones asociadas y las consecuencias de este para la vida.

En correspondencia Ouellette et al., (30) en el estudio reciente, refieren que la pérdida de un riñón trasplantado es como una perturbación profunda e intrusiva en la vida, similar a la experiencia inicial de la diálisis. Los niños y sus familias viven con la incertidumbre del rechazo de riñón y otras complicaciones médicas. El rechazo del injerto y la pérdida reflejan un deterioro del funcionamiento físico y está asociado al sufrimiento psicoemocional. Aunque los niños pueden experimentar incertidumbre y devastación si se produce un fallo del injerto que tiene un trasplante de riñón, el trasplante también introduce indirectamente nuevos retos. Según Jana et al., (31) la depresión en los niños se asocia con baja calidad de vida y la ansiedad se asocia con las hospitalizaciones frecuentes, rechazos y complicaciones.

ISSN-PRINT

1794-9831

E-ISSN 2322-7028

Vol. 17 No. 3

Sep - Dic 2020

Cúcuta, Colombia 
Igual que en nuestro estudio, refiere Ramírez (32), el cuidador manifiesta temor frente al rechazo o la pérdida del riñón. Se angustia frente a volver a empezar o regresar a la diálisis . De otra parte, Grenda (33) relata que manejar las complicaciones del rechazo es un asunto indeseable después del trasplante, cuya incidencia varía según el tipo de donación y varios factores de riesgo clínicos e inmunológicos que lleva a la evaluación diaria del nivel de creatinina sérica, así como los estudios de imagen del riñón y el flujo sanguíneo con ecografía, y algunos centros realizan biopsias renales (33).

\section{Conclusiones}

- Gran parte del éxito del trasplante se le atribuye al cuidador, pues es quien cuida permanentemente del niño y quien guía los cuidados, ya que la condición de ser niños les otorga total dependencia del cuidador.
- Los cuidadores de los niños trasplantados, generalmente, son mujeres y madres cabeza de familia y son quienes ayudan a alcanzar los objetivos del tratamiento pre, trans y postrasplante renal, esenciales para la supervivencia del niño. Así, los medicamentos y el cuidador son la vida del trasplante.

- Las emociones y el entorno familiar son fundamentales en la trayectoria del niño con trasplante renal, desde el descubrimiento de la enfermedad, existen asuntos positivos, negativos y de incertidumbre, como lo es el rechazo, este es un llamado de atención a la hora de llevar a cabo el cuidado del niño trasplantado. Asimismo, la vida del niño, cuidador y familia y en general la calidad de vida, se ven modificados con el trasplante.

\section{Conflicto de Intereses}

Los autores declaran no tener ningún conflicto de intereses.

\section{Referencias bibliográficas}

1. Fernández-Camblor $\mathrm{C}$, Melgosa-Hijosa M. Enfermedad renal crónica en la infancia. Diagnóstico y tratamiento. Protoc diagn ter pediatr. 2014; 1:385-401.

2. García-Meseguer C, Vila-Santandreu A. Trasplante renal pediátrico. Protoc diagn ter pediatr. 2014;1:435-53.

3. Riva N, Cáceres-Guido P, Rousseau M, Dip M, Monteverde M, Imventarza O, et al. Farmacovigilancia de inhibidores de calcineurina en trasplante renal y hepático pediátrico. Farm Hosp. [Internet]. 2013 Dic [citado 2019 Oct 22]; 37(6):441-449. Disponible en: http://dx.doi.org/10.7399/FH.2013.37.6.778

4. Moreso F, Hernández D. ¿Ha mejorado la supervivencia del injerto tras el trasplante renal en la era de la moderna inmunosupresión? Nefrología (Madr.) [Internet]. 2013 [citado 2019 Oct 22]; 33(1):14-26. Disponible en: http://scielo.isciii.es/scielo.php?script=sci_arttext\&pid $=$ S0211-69952013000100003\&lng $=$ es

5. Ruiz-Calzado MR. La adherencia terapéutica en el paciente trasplantado renal. Enferm Nefrol [Internet]. 2013 Dic [citado 2019 Oct 22]; 16(4):258-270. Disponible en: http:// dx.doi.org/10.4321/S2254-28842013000400007

6. Fernández-Rojas S. Sobrevida en trasplante renal en el Hospital Nacional de Niños. Acta méd. costarric [Internet]. 2019 June [cited 2019 Oct 22]; 61(2):62-67. Available from: http:// www.scielo.sa.cr/scielo.php?script $=$ sci arttext\&pid $=$ S0001-60022019000200062\&1$\underline{\mathrm{ng}}=\mathrm{en}$

7. Verghese PS. Pediatric kidney transplantation: a historical review. Pediatr Res 2017; 81(1): 259-264. https://doi.org/10.1038/pr.2016.207

8. República de Colombia. Municipio de Medellín. Acuerdo_27 de 2015. Gaceta Oficial No4340. [internet] Medellín; Noviembre 3 de 2015 [acceso noviembre 9 de 2016] disponible en: https://www.medellin.gov.co/irj/go/km/docs/pccdesign/SubportaldelCiudadano_2/PlandeDesarrollo_0 15/Publicaciones/Shared\%20Content/GACETA\%20OFI- 


\section{CIAL/2015/Gaceta\%204340/ACUERDO\%200027\%20DE\%202015.pdf}

9. Grove S, Gray J, Burns N. Investigación en enfermería: Desarrollo de la práctica enfermera basada en la evidencia, 6 ed. Barcelona: Elsevier. 2016

10. Corbin J, Strauss A. Basics of qualitative research. Techiques and procedures for developing grounded theory. 4 ed. Los Ángeles: SAGE. 2015

11. Ritzer G. Interaccionismo simbólico. En: teoría sociológica moderna. Madrid: McGrawHill. 2002,247-287

12. Otzen T, Manterola C. Técnicas de Muestreo sobre una Población a Estudio. Int. J. Morphol. [Internet]. 2017 Mar [citado 2019 Oct 22]; 35(1):227-232. Disponible en: http:// dx.doi.org/10.4067/S0717-95022017000100037

13. Organización Panamericana de la Salud, Consejo de Organizaciones Internacionales de las Ciencias Médicas. Pautas éticas internacionales para la investigación relacionada con la salud con seres humanos. Cuarta edición [Internet] Ginebra: Consejo de Organizaciones Internacionales de las Ciencias Médicas; 2017 [Consultado 2016 Sep 18] Disponible en: https://cioms.ch/wpcontent/uploads/2017/12/CIOMS-EthicalGuideline_SP INTERIOR-FINAL.pdf

14. Emanuel E. ¿Qué hace que la investigación clínica sea ética? Siete requisitos éticos. En: Lolas S F, Quezada S A. Pautas Éticas de Investigación en Sujetos Humanos: Nuevas Perspectivas. [Internet] Santiago de Chile: Organización Panamericana de la Salud \& Organización Mundial de la Salud; 2003: 83-95 [Actualización 2016 Nov 3; consultado 2016 Oct 01] Disponible en: https://www.uach.cl/uach/_file/5-5b33b10f6e7c6.pdf

15. República de Colombia. Ministerio de Salud. Resolución 8430 de 1983, octubre 4, por la cual se establecen las normas científicas, técnicas y administrativas para la investigación en salud. [Internet] Bogotá: El Ministerio; 1983 [Consultado 2016 Sep 3]. Disponible en: https://www.minsalud.gov.co/sites/rid/Lists/BibliotecaDigital/RIDE/DE/DIJ/RESOLUCION-8430-DE-1993.PDF

16. Noreña AL, Alcaraz-Moreno N, Rojas JG, Rebolledo-Malpica D. Aplicabilidad de los criterios de rigor y éticos en la investigación cualitativa. Aquichán [Internet]. 2012 Dec [cited 2019 Oct 21]; 12(3):263-274. Available from: http://www.scielo.org.co/scielo. php?script $=$ sci_arttext\&pid $=$ S1657-59972012000300006\&lng=en

17. Medellín-Olaya J, Carrillo-González GM. Soporte social percibido y calidad de vida de personas con enfermedad renal crónica sometidas a trasplante renal. av.enferm 2014; 32(2):206-216. https://doi.org/10.15446/av.enferm.v32n2.46099

18. Goreti-da-Silva CM, Celina D, Bartira-de-Aguiar R, Vieira-da-Silva OC, de-Moraes H. Family experience in the kidney transplant process from a living donor. Acta paul. enferm. [Internet]. 2015 June [cited 2019 Oct 19]; 28(3): 275-280. Available from: http:// dx.doi.org/10.1590/1982-0194201500046

19. DePasquale N, Cabacungan A, Ephraim PL, Lewis-Boyér LP, Powe NR, Boulware LE. Family Members' Experiences With Dialysis and Kidney Transplantation. Kidney Med 2019; 1(4): 171-179. https://doi.org/10.1016/j.xkme.2019.06.001

20. Rodríguez-Rodríguez T, Fonseca-Fernández M. Guía de atención psicológica a pacientes y familiares en el proceso de donación y trasplante de órganos. MediSur [Internet] 2015 [consultado 19 de octubre de 2019]; 13(4):560-568. Disponible en: http://scielo.sld.

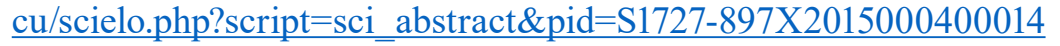

21. Tejada-Ortigosaa EM, Flores-Rojasb K, Moreno-Quintanaa L, Gil-Camposb M. Perspectiva de género sobre los cuidados informales de las niñas y niños con enfermedades raras. An Pediatr (Barc), 2019; 91(4):281-282. https://doi.org/10.1016/j. anpedi.2019.03.007

22. Fernández AM, Agüera-Morales M, Muñoz-Cepeda MÁ, González-Roncero FM, Lorenzo I, Mendiluce-Herrero A, et al. Monitorización farmacológica de inmunosupreso- 
ISSN-PRINT

$1794-9831$

E-ISSN 2322-7028

Vol. 17 No. 3

Sep - Dic 2020

Cúcuta, Colombia res. Nefrología 2016; 7(S2): 51-6.

23. Pinto-Afanador N. Bienestar Espiritual de los cuidadores familiares de niños que viven enfermedad crónica. Rev. invest. enfer. 2007; 9(1):20-35.

24. Davis KG. Integrating Pediatric Palliative Care into the School and Community. Pediatr Clin North Am 2016;63(5):899-911. https://doi.org/10.1016/j.pcl.2016.06.013

25. Agredo-Lucio MA, Santamaría-Suárez MP, Restrepo-Restrepo JM. Transición en adolescentes con trasplante renal: El paso de atención pediátrica al cuidado de adultos. Rev. Colomb. Nefrol. 2016; 3(2):120-123. http://doi.org/10.22265/acnef.3.2.244

26. Carrillo-Algarra AJ, Mesa-Melgarejo L, Moreno-Rubio F. El cuidado en un programa de trasplante renal: un acompañamiento de vida. Aquíchan. 2015; 15(2):271-282. http://doi. org/10.5294/aqui.2015.15.2.10

27. Navarrete B, Alvarado R. La enfermedad renal crónica en el niño y el cuidado de enfermería. Rev. Colomb. Enferm. [Internet] 2013 [consultado 2018 May 2]; 8(8):175-86. Disponible en: http://revistacolombianadeenfermeria.unbosque.edu.co/article/view/557

28. Barra-Barrueta O, Morillo-Verdugo R. Grupo de Adherencia Terapéutica ADHEFAR de la SEFH. Lo que debes saber sobre la adherencia al tratamiento. Sociedad Española de Farmacia Hospitalaria (SEFH) y Boehringer Ingelheim. [internet] 2017 [acceso 21 de octubre de 2019]. Disponible en: https://www.sefh.es/bibliotecavirtual/Adherencia2017/ libro ADHERENCIA.pdf

29. American Society of Transplantation. Bartosh S, Dipchand A, Chavers B. Trasplante renal pediátrico. Una guía para pacientes y familias [Internet] 2016 [acceso octubre 1 de 2019] disponible en: https://www.myast.org/sites/default/files/SPANISH_Pediatric $\% 20$ Kidney\%20Transplantation.pdf

30. Ouellette A, Achille M, Paquet M. The experience of kidney graft failure: Patients perspectives. Qual Health Res. 2009 Ago; 19(8):1131-38. http://doi.org/10.1177/1049732309341733

31. Jana AK, Sircar D, Waikhom R, Praharaj SK, Pandey R, Ray Chaudhury A, Dasgupta S. Depression and anxiety as potential correlates of post-transplantation renal function and quality of life. Indian J Nephrol. 2014 Sep-Oct; 24(5): 286-290. http://doi. org/10.4103/0971-4065.132996

32. Ramírez CA. Aprender a vivir con un órgano trasplantado. Rev. cienc. cuidad. 2019; 16(3): 93-102. https://doi.org/10.22463/17949831.1596

33. Grenda R. Delayed graft function and its management in children. Pediatr Nephrol 2017; 32(7):1157-1167. https://doi.org/10.1007/s00467-016-3528-9 